\title{
Radiometric measurement requirements to derive information on phytoplankton community composition from satellite
}

\author{
Peter Gege
}

DLR, Remote Sensing Technology Institute,

Oberpfaffenhofen, 82234 Wessling, Germany

IEEE IGARSS 2021, Brussels, Belgium, 11-16 July 2021

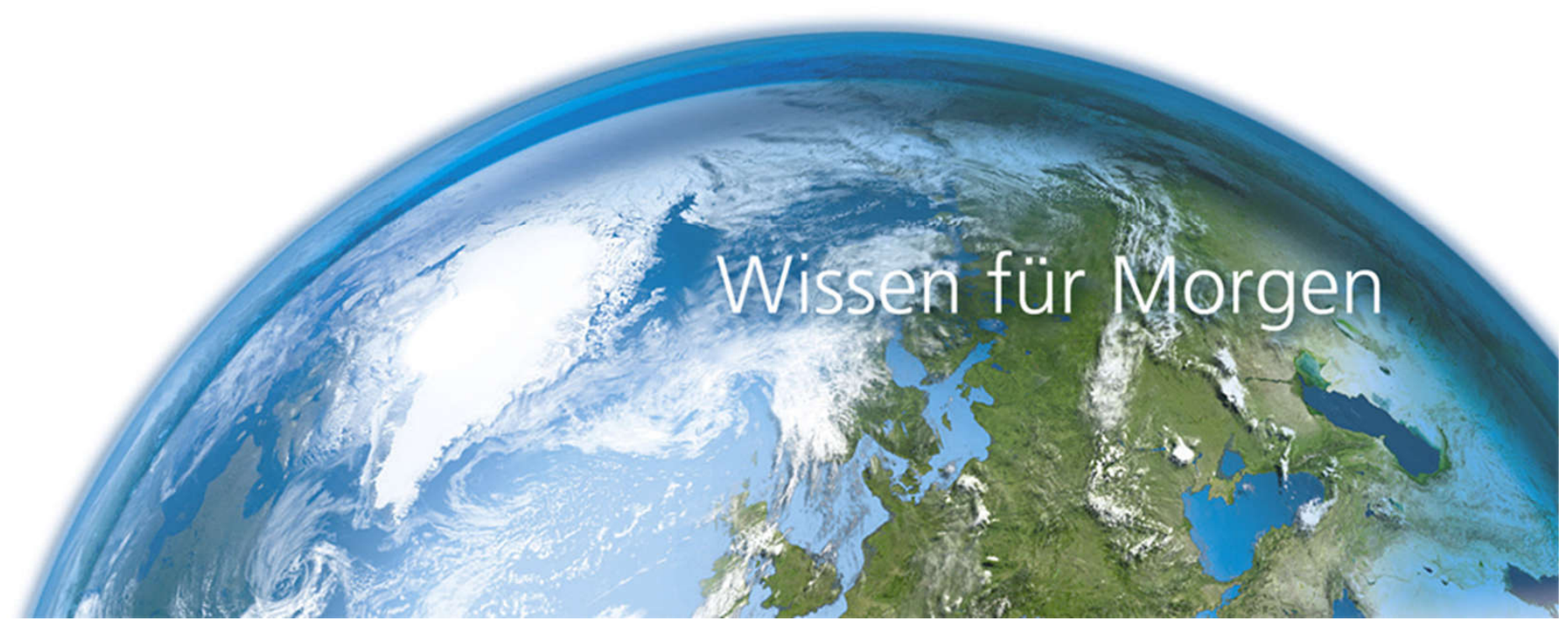




\section{Introduction}

CEOS study ${ }^{1}$

$>$ requirements for satellite system dedicated to inland and coastal waters

sensitivity analysis for spectral and radiometric requirements with focus on chl-a

Sensitivity analysis has been complemented for phytoplankton classification

assessment of the potential of multi- and hyperspectral sensors

$>$ design of new sensors

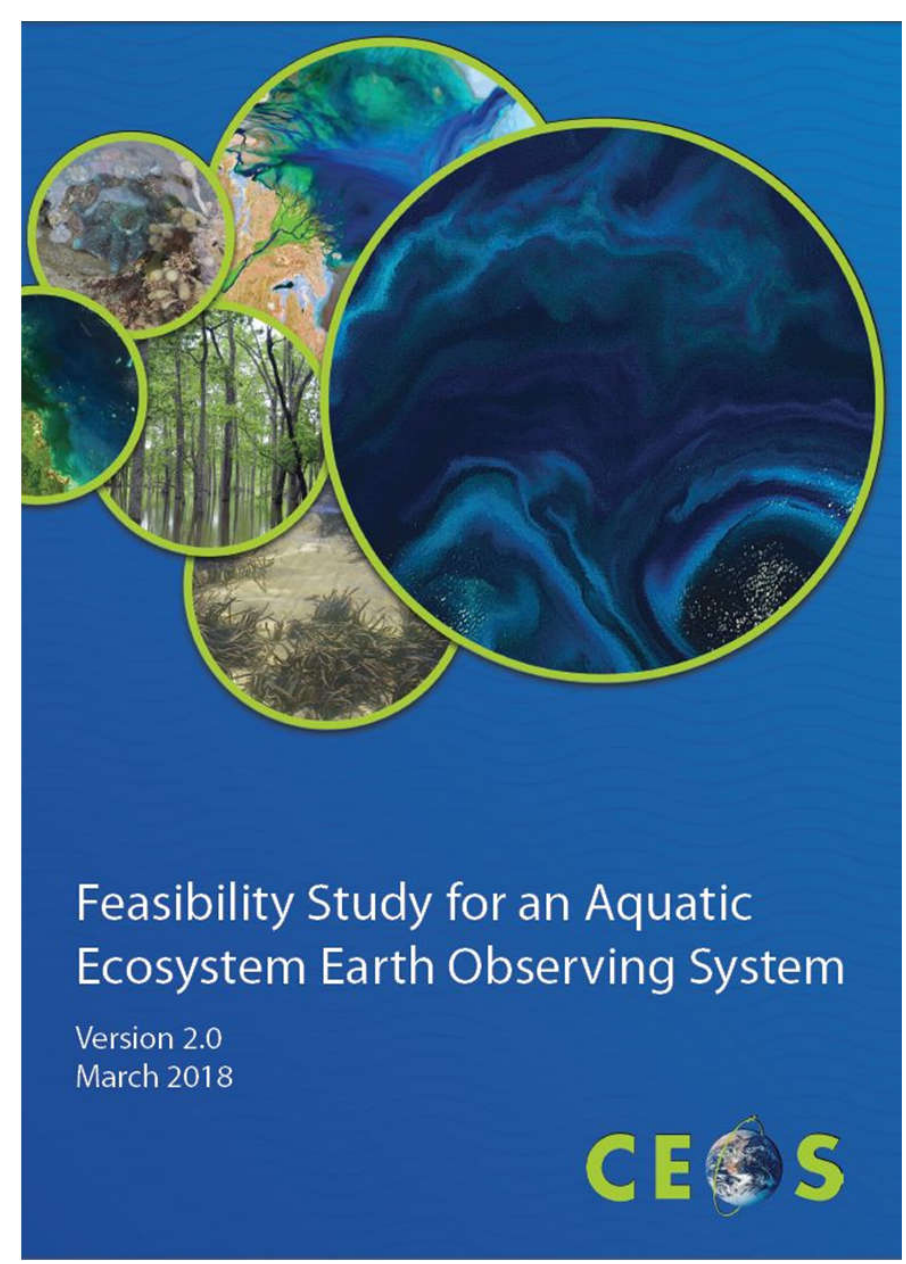

1https://ceos.org/document management/Publications/Feasibility-Study-for-an-Aquatic-Ecosystem-EOS-v.2-hires 05April2018.pdf
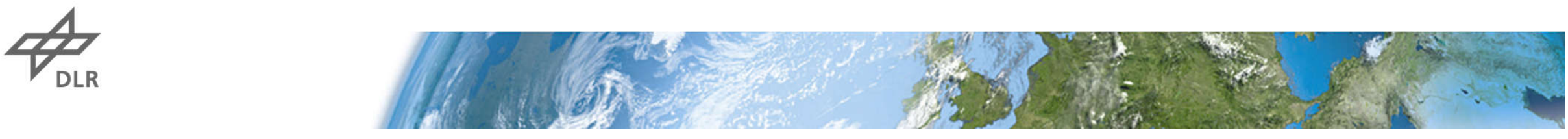


\section{Simulations at bottom of atmosphere: modeling}

- Bio-optical model ${ }^{1}$ simulates remote sensing reflectance, $R_{r s}(\lambda)$

- Phytoplankton community composition is represented by 4 absorption spectra ${ }^{2}$
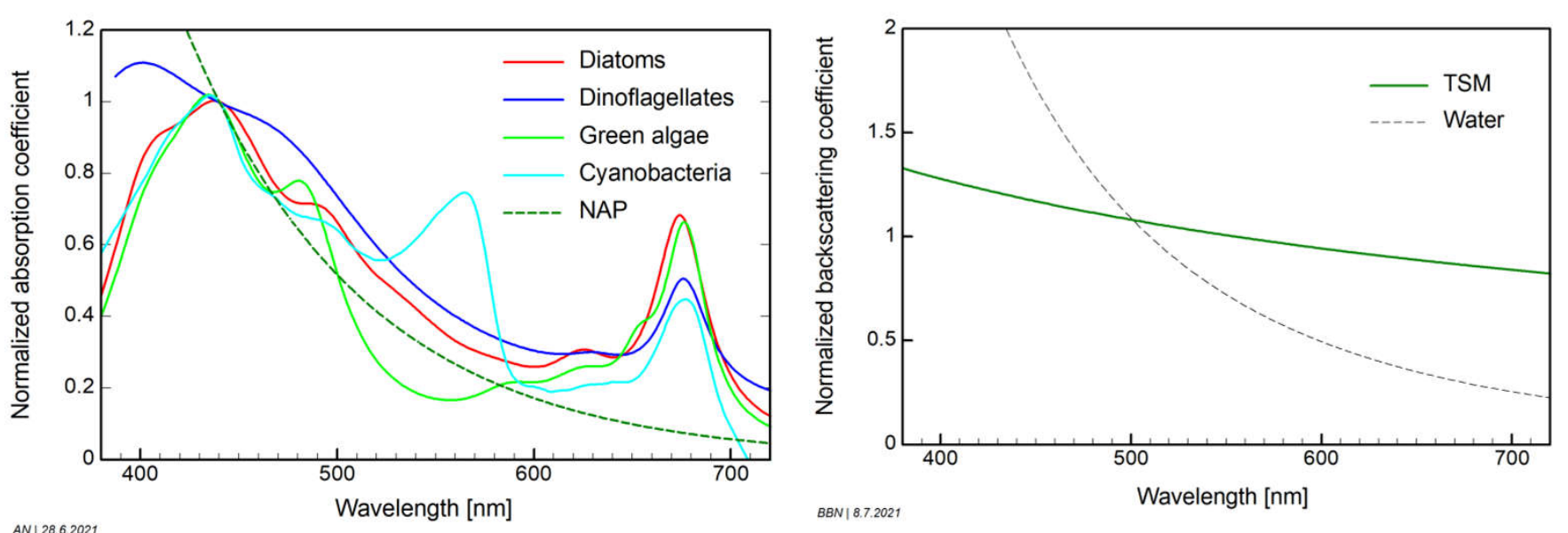

$$
\begin{aligned}
& a_{\text {dia }}^{*}(440)=0.036 \mathrm{~m}^{2} \mathrm{mg}^{-1} \\
& a_{\text {dino }}^{*}(440)=0.050 \mathrm{~m}^{2} \mathrm{mg}^{-1} \\
& a_{\text {green }}^{*}(440)=0.035 \mathrm{~m}^{2} \mathrm{mg}^{-1} \\
& a_{c y a}^{*}(440)=0.033 \mathrm{~m}^{2} \mathrm{mg}^{-1} \\
& a_{N A P}^{*}(440)=0.027 \mathrm{~m}^{2} \mathrm{~g}^{-1} \\
& b_{b, N A P}^{*}(555)=0.011 \mathrm{~m}^{2} \mathrm{~g}^{-1} \\
& b_{b, p h y}=0 \text { (retrieval based solely } \\
& \text { on phytoplankton absorption) }
\end{aligned}
$$

- Impact of chl-a concentration, $C:\left|\Delta R_{r s, C}(\lambda)\right|=\left|R_{r s}(\lambda, 1.3 C)-R_{r s}(\lambda, C)\right|$

- Impact of phytoplankton group: $\left|\Delta R_{r s, i, j}(\lambda)\right|=\left|R_{r s}\left(\lambda, a_{i}^{N}(\lambda)\right)-R_{r s}\left(\lambda, a_{j}^{N}(\lambda)\right)\right|$

- Signal-to-noise ratio: $S N R^{B O A}(\lambda)=\frac{R_{r s}(\lambda)}{\left|\Delta R_{r S}(\lambda)\right|}+\frac{\rho L_{s k y}(\lambda)}{E_{d}(\lambda)\left|\Delta R_{r S}(\lambda)\right|}$

${ }^{1}$ Albert, A.; Mobley, C.D. An analytical model for subsurface irradiance and remote sensing reflectance in deep and shallow case-2 waters. Opt. Express 2003, 11, 2873-2890.

${ }^{2}$ Courtesy M. Hieronymi, HZG Geesthacht, Germany, for the cyanobacteria spectrum
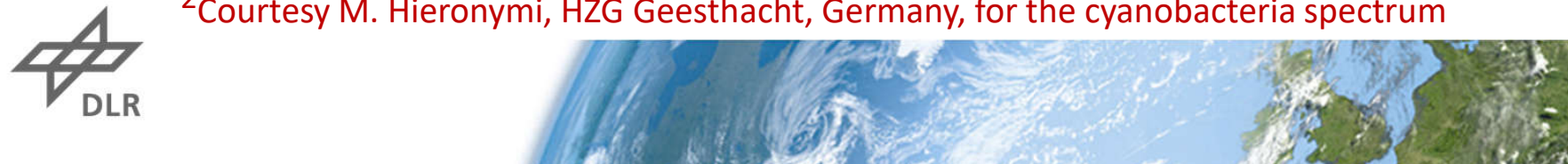


\section{Simulations at bottom of atmosphere: scenarios}

- Covered ranges: Chl-a 0.05-100 mg m ${ }^{-3}$, TSM 0.2-30 g m m-3 aCDOM(440) 0.01-5 m-1

- Concentration combinations are oriented on well-studied waters („scenarios")

- 1000 concentration combinations per phytoplankton group per scenario

\begin{tabular}{|l|c|c|c|c|c|c|c|}
\hline Scenario & C- & C+ & X- & X+ & Y- & Y+ \\
\hline Represents & Low chl-a & High chl-a & Low TSM & High TSM & Low CDOM & High CDOM \\
\hline Example & Reef water & Finnish lakes & Lake Constance & Netherlands & Lake Garda & Lake Peipsi \\
\hline$C, \mathrm{mg} \mathrm{m}^{-3}$ & $0.05-0.2$ & $10-100$ & $0.5-10$ & $10-50$ & $0.2-10$ & $1-20$ \\
\hline$X, \mathrm{~g} \mathrm{~m}^{-3}$ & $1-2$ & $5-15$ & $0.5-5$ & $10-30$ & $0.2-20$ & $1-10$ \\
\hline$Y, \mathrm{~m}^{-1}$ & $0.01-0.1$ & $1.5-4$ & $0.1-1$ & $0.5-1.5$ & $0.04-1$ & $1-5$ \\
\hline
\end{tabular}
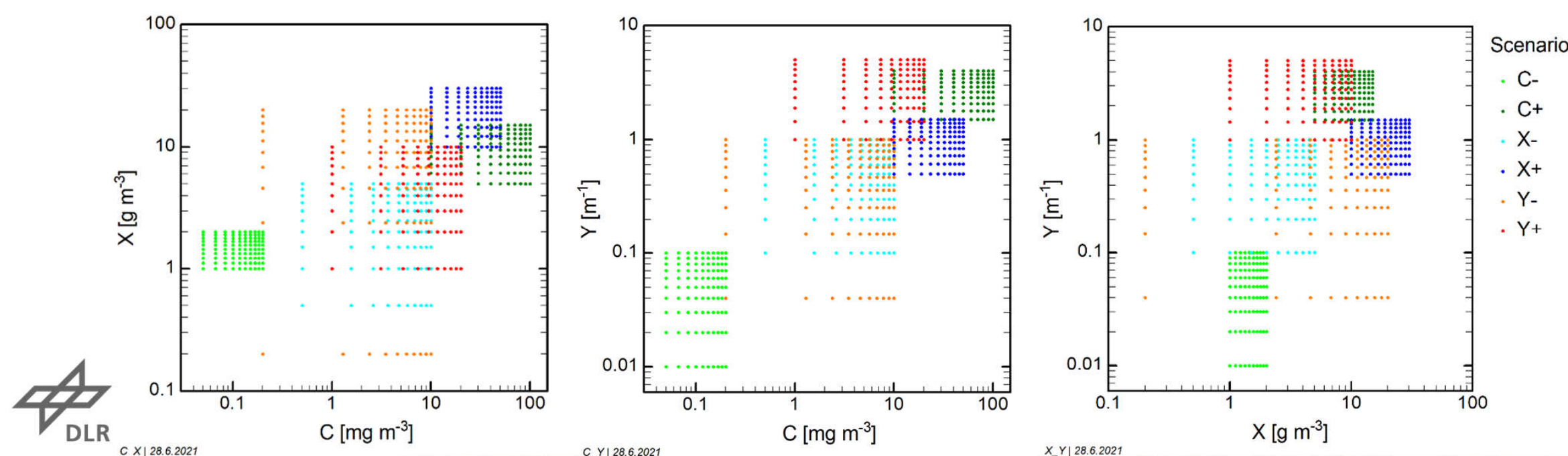

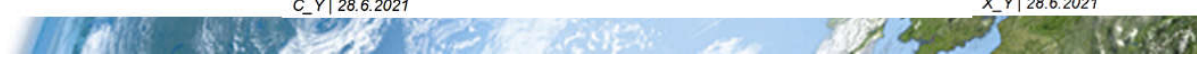




\section{Simulations at top of atmosphere}

- $L^{T O A}(\lambda)=L^{\text {path }}(\lambda)+t_{A}(\lambda)\left[R_{r s}(\lambda) E_{d}(\lambda)+\rho L_{s k y}(\lambda)\right]$

- $\left|\Delta L^{T O A}(\lambda)\right|=t_{A}(\lambda) E_{d}(\lambda)\left|\Delta R_{r s}(\lambda)\right|$

- $\quad \operatorname{SNR}^{T O A}(\lambda)=\frac{L^{T O A}(\lambda)}{\left|\Delta L^{T O A}(\lambda)\right|}=\frac{L^{\text {path }}(\lambda)}{t_{A}(\lambda) E_{d}(\lambda)\left|\Delta R_{r s}(\lambda)\right|}+\operatorname{SNR}^{B O A}(\lambda)$

- Modtran-6 simulates $L^{\text {path }}(\lambda), t_{A}(\lambda), E_{d}(\lambda)$ and $L_{s k y}(\lambda)$

- Environmental conditions: mid-latitude summer atmosphere, horizontal visibility $50 \mathrm{~km}$, sun zenith angle of $40^{\circ}$

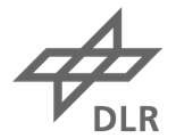




\section{Results: SNR required at TOA due to path radiance}

$$
\operatorname{SNR}^{\text {path }}(\lambda)=\frac{L^{\text {path }}(\lambda)}{t_{A}(\lambda) E_{d}(\lambda)\left|\Delta R_{r s}(\lambda)\right|}
$$

Minimum useable wavelength for SNR $=800^{1}$

\begin{tabular}{|l|c|c|c|c|c|}
\hline$\left|\Delta \boldsymbol{R}_{\boldsymbol{r s}}\right|$ & \multicolumn{5}{|c|}{ Sun zenith angle } \\
\hline & $\mathbf{0}^{\circ}$ & $\mathbf{2 0 ^ { \circ }}$ & $\mathbf{4 0 ^ { \circ }}$ & $\mathbf{6 0}$ & $\mathbf{7 0}^{\circ}$ \\
\hline $\mathbf{1 0 ^ { - 4 }} \mathbf{s r}^{-1}$ & 438 & 441 & 448 & 469 & 494 \\
\hline $\mathbf{1 0}^{-\mathbf{5}} \mathbf{s r}^{-1}$ & 740 & 740 & 743 & 769 & 785 \\
\hline
\end{tabular}
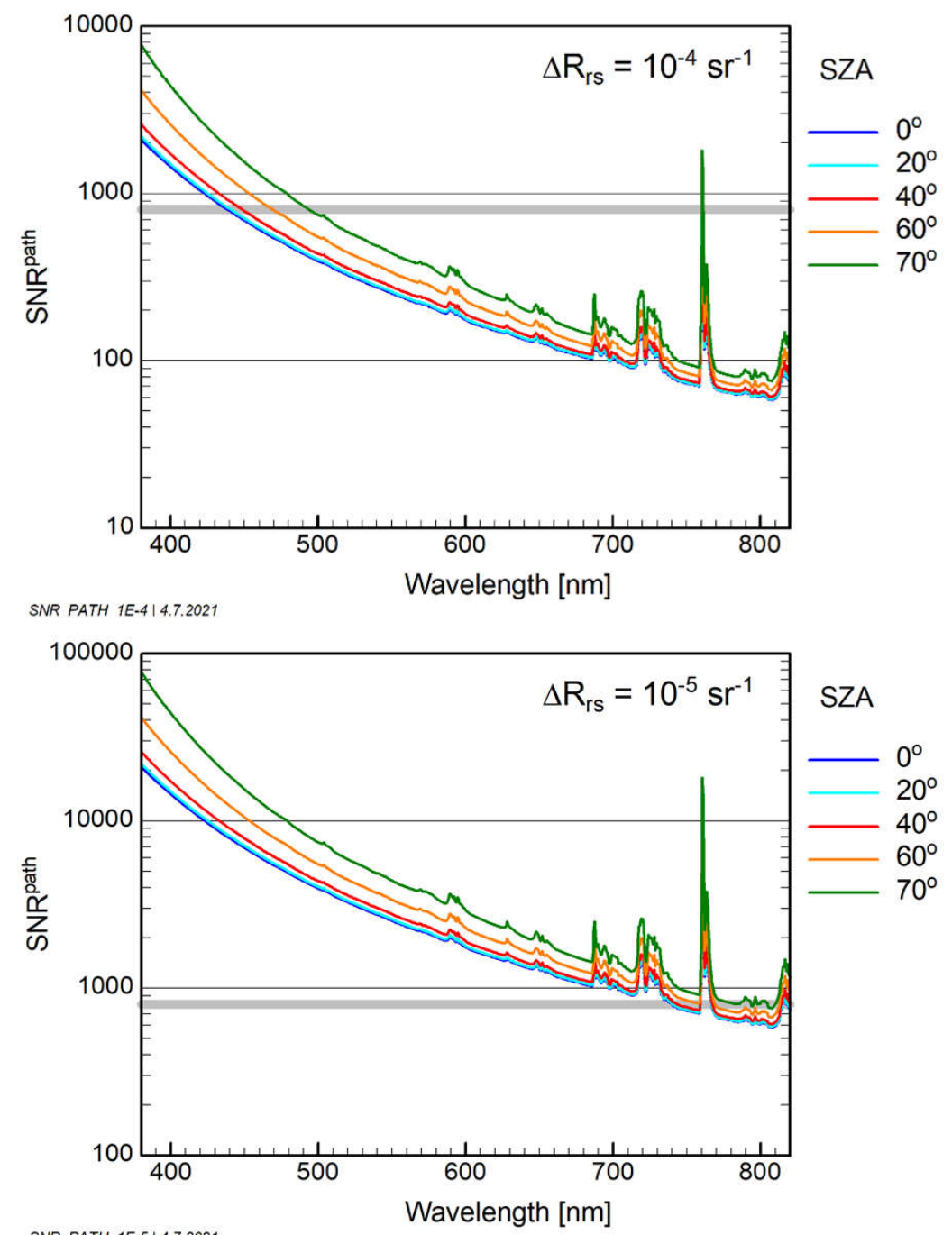

${ }^{1}$ Recommended by Muller-Karger et al. 2018

https://esajournals.onlinelibrary.wiley.com/doi/10.1002/eap.1682

Plots adopted from: Gege, P.; Dekker, A.G. Spectral and radiometric measurement requirements for inland, coastal and reef waters.

Remote Sensing 2020, 12, 2247. doi:10.3390/rs12142247
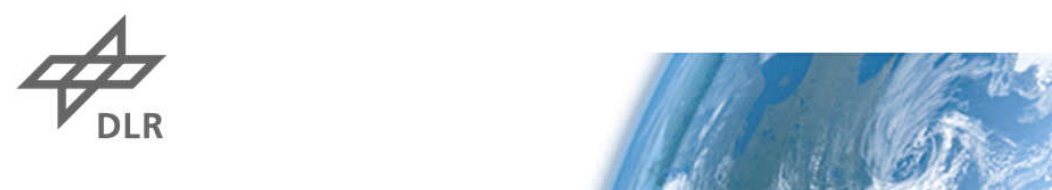


\section{Results: Measurement requirements at TOA}

Resolve $30 \%$ change of chl-a concentration

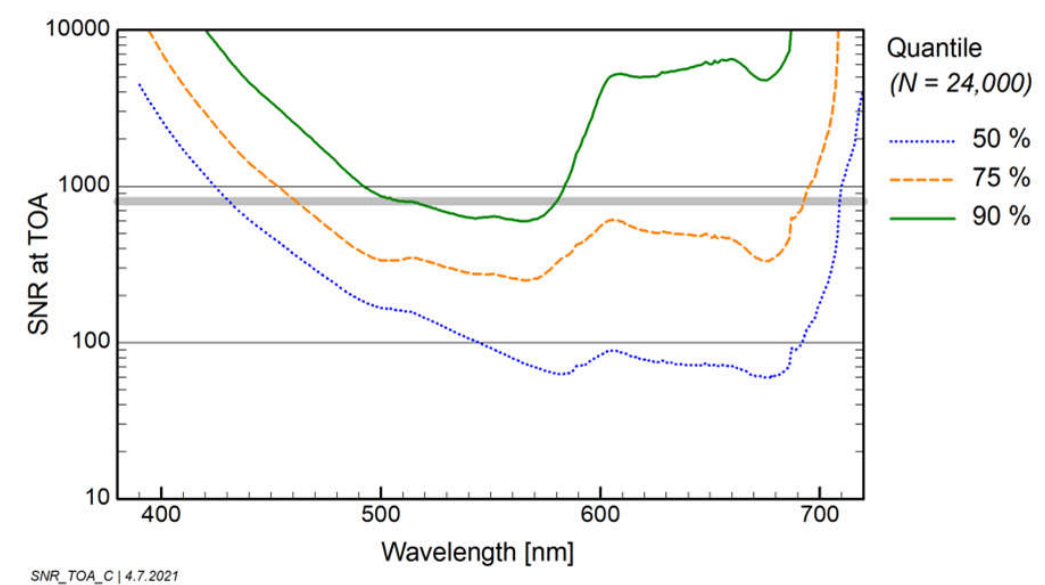

- Useable wavelengths for $\mathrm{SNR}=800^{1}$

\begin{tabular}{|c|c|c|}
\hline Quantile & From [nm] & To [nm] \\
\hline $\mathbf{5 0 \%}$ & 407 & 618 \\
\hline $\mathbf{7 5} \%$ & 431 & 606 \\
\hline $\mathbf{9 0} \%$ & 507 & 580 \\
\hline
\end{tabular}

\section{Resolve exchange of phytoplankton group}

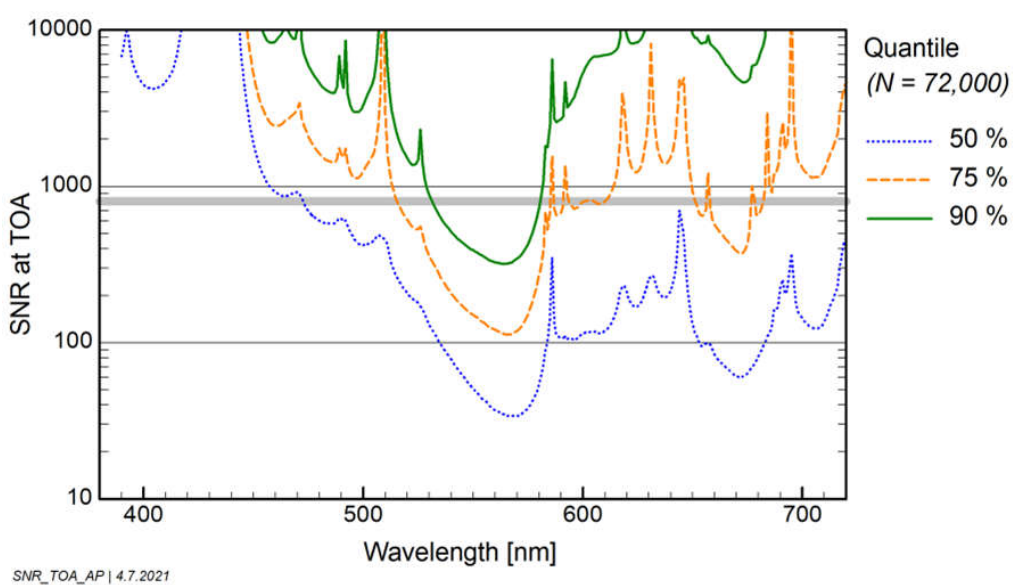

- Useable wavelengths for SNR $=800^{1}$

\begin{tabular}{|c|c|c|}
\hline Quantile & From [nm] & To [nm] \\
\hline $\mathbf{5 0 \%}$ & 440 & 728 \\
\hline $\mathbf{7 5} \%$ & 515 & 585 \\
\hline & 650 & 682 \\
\hline $\mathbf{9 0} \%$ & 531 & 581 \\
\hline
\end{tabular}

${ }^{1}$ Recommended by Muller-Karger et al. 2018

https://esajournals.onlinelibrary.wiley.com/doi/10.1002/eap.1682 


\section{Summary}

Required SNR at TOA

$>$ Dominated by path radiance

$>$ Depends strongly on wavelength; SNR $>1000$ below $500 \mathrm{~nm}$ for $\left|\Delta R_{r S}\right|<10^{-4} \mathrm{sr}^{-1}$

$>$ Chl-a changes of $30 \%$ : SNR > 600 above $460 \mathrm{~nm}$ for $75 \%$ quantile

$>$ Phytoplankton classification: SNR > 800 above $500 \mathrm{~nm}$ for $75 \%$ quantile

A paper about these simulations is in preparation. It includes additionally sensor requirements (NEL).

\section{Thank you for your attention!}
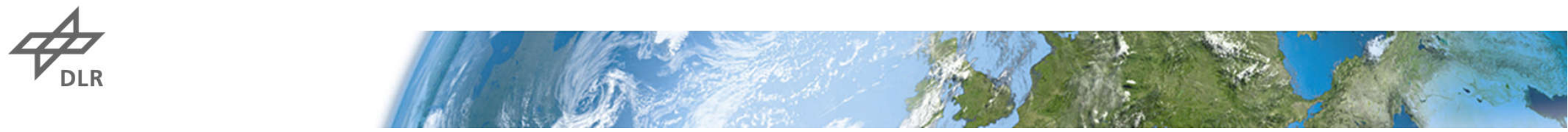